\title{
A Linear Relaxation Method for Computing Workspace Slices of the Stewart Platform
}

\author{
Oriol Bohigas, Montserrat Manubens, and Lluís Ros \\ Institut de Robòtica i Informàtica Industrial (CSIC-UPC) \\ 08028 Barcelona, Catalonia \\ Email: \{obohigas,Ilros,mmanuben\}@iri.upc.edu
}

\begin{abstract}
The workspace of a Stewart platform is a complex sixdimensional volume embedded in the Cartesian space defined by six pose parameters. Because of its large dimension and complex shape, this volume is difficult to compute and represent, and comprehension on its structure is being gained by studying its three-dimensional slices. While successful methods have been given to determine the constantorientation slice, the computation and appropriate visualization of the constant-position slice (also known as the orientation workspace) has proved to be a challenging task. This paper presents a unified method for computing both of such slices, and any other ones defined by fixing three pose parameters, on general Stewart platforms possibly involving mechanical limits on the active and passive joints. Advantages over existing methods include, in addition to the previous, the ability to determine all connected components of the workspace, and any motion barriers present in its interior.
\end{abstract}

\section{Introduction}

Due to their advantages in terms of dynamic properties, load-carrying capacity, high accuracy, and stiffness, parallel manipulators are widely used as flight simulators [1], highprecision positioning devices [2,3], vibration isolators [4], machining centers [5,6], or surgical robots [7]. The assembly constraints imposed by their kinematic design, however, substantially reduce the set of poses that such manipulators can attain, leading to highly-constrained workspaces in most of the cases. The availability of proper tools to accurately compute and represent such workspaces is thus of utmost importance, not only to assist the robot designer during the conception of the platform, but also to be able to implement trajectory planners more efficiently [8], once an adequate design has been chosen for a particular application.

The workspace of the Stewart platform, in particular, is hard to compute and visualize [9]. Its large dimension and complex shape, which may encompass several connected components, difficult any attempt of computing it exhaustively. In many situations, fortunately, the platform either operates with a fixed orientation or rotates about a fixed point, so that it can be assumed that three of the six pose parameters are held constant, leading to three-dimensional workspaces that are easier to obtain and represent. The constant-orientation workspace, in particular, is clearly understood, and fast geometric algorithms exist for computing its boundary [10], even in the presence of joint limits in the passive joints, or potential link-link interferences [11]. Interval analysis methods have also been given to compute the interior of such workspace [12]. The constant-position workspace, also known as the orientation workspace [13], has also been studied, but its computation and visualization turn out to be more problematic, due to the complexity of the intervening equations, and to the difficulty of representing orientations in an intuitive way. Previous methods either assume one of the orientation angles held fixed [14,15], thus producing two-dimensional sections of the workspace only, or let the three angles vary [13,16-19], but all methods rely on some sort of discretization, which leads to incomplete or less accurate output in some situations. The most recent of such methods is fast, and provides appealing visualizations of the orientation workspace [19], but mechanical limits in the passive joints are neglected, so that the computed workspace is, actually, an overestimation of the real workspace. Another limitation of this method, as well as of those in $[16,18]$, is that it only allows obtaining the workspace component that is achievable from a known configuration, which provides only a partial picture of the whole motion range of the manipulator.

Despite the literature on the topic is rich, three important requirements are not fully met by previous approaches. First of all, a method should ideally be complete, i.e., it should be able to obtain all connected components of the workspace, rather than just one achievable from a given configuration. Such ability is useful to the robot designer, to choose the appropriate component on which to assemble the manipulator, according to the operational volume desired, or to motion restrictions imposed by the application environment. Moreover, the method should be accurate, not only providing a precise representation of the workspace volume, but also of any motion barriers interior to the volume. Such barriers constitute true obstacles within the workspace, and may be encountered on Stewart platforms of special geom- 
etry, as shown in the paper. Finally, the method should be as general as possible, allowing to determine the constantorientation and constant-position workspaces, and any other of the twenty workspaces that can be defined by fixing three of the six pose parameters of the platform, in order to understand the motion capability of the platform under any of the corresponding working modes.

The continuation approach in $[16,20]$ and references therein is perhaps the closest to satisfy the previous requirements simultaneously. This approach uses ray-shooting techniques in conjunction with continuation methods to track the configurations for which the moving platform loses some of its instantaneous degrees of freedom, which yield the boundary of the workspace and its interior barriers when properly analyzed. However, while this approach is general and accurate in favorable cases, it loses boundary segments in several situations, as mentioned in $[21,22]$, and therefore it is not complete.

A method satisfying the three previous requirements is given in this paper, valid for Stewart platforms of arbitrary geometry, involving mechanical limits both on the active and passive joints. The approach is similar to the one given in [22] for general structure manipulators, but specificities of the Stewart platform are herein exploited to achieve a much faster computation of the workspace. The method entails formulating a system of equations defining the boundary of the workspace (Section 2), and then using an iterative procedure based on linear relaxations [23,24] to isolate slices of the boundary exhaustively at the required resolution (Section 3). It is worth noting that while no consensus has been reached as to how should platform orientations be parameterized in order to yield intuitive workspace representations, the method we propose here does not adopt any particular choice on this respect, and can obtain the orientation workspace under any possible parameterization, including those based on conventional Euler angles [19], tilt-andtorsion angles [13,25], or Euler-Rodrigues parameters [17]. The approach has been implemented and validated succesfully on several test cases (Section 4) and possible applications and extensions have been identified (Section 5).

\section{Formulation}

A Stewart platform consists of a body (the platform) linked to the ground (the base) by means of six legs, where each leg is a universal-prismatic-spherical chain (Fig. 1). The most general version of such platform follows the so-called 6-6 UPS design, where the leg anchor points are all different, though not necessarily coplanar [26]. The six prismatic joints are active, i.e. actuated, allowing to control the six degrees of freedom of the platform, and the remaining joints are passive.

Let $O X Y Z$ and $P X^{\prime} Y^{\prime} Z^{\prime}$ be fixed and moving reference frames, respectively attached to the base and the platform (Fig. 1). Any pose of the platform can be uniquely represented by a $3 \times 3$ rotation matrix $\mathbf{R}$ that provides the orientation of $P X^{\prime} Y^{\prime} Z^{\prime}$ relative to $O X Y Z$, and the position vector $\mathbf{p}=[x, y, z]^{\top}$ of point $P$ in the fixed frame. Not all values

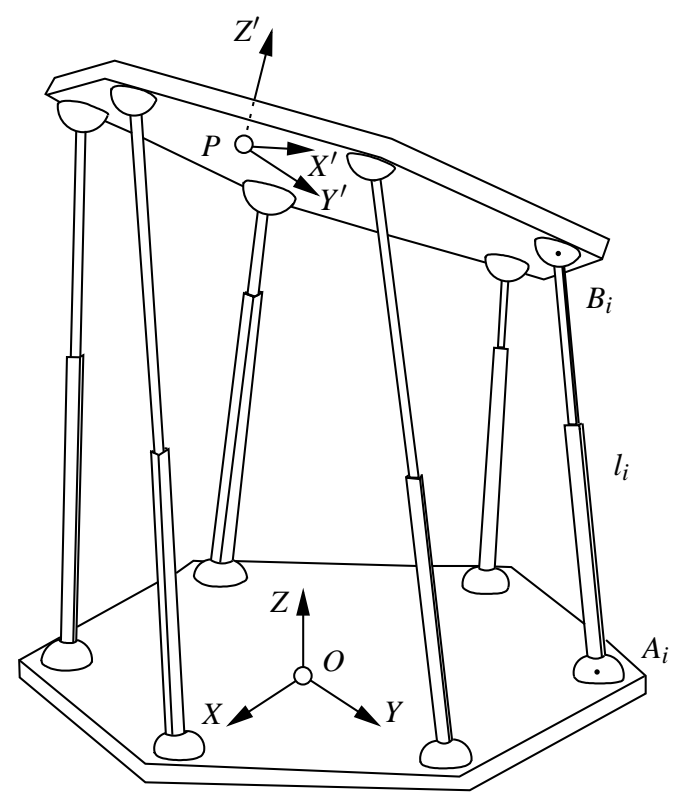

Fig. 1. A Stewart platform. The base and platform joints are meant to be universal and spherical, respectively.

for $\mathbf{R}$ and $\mathbf{p}$ are allowed, however, because the mechanical limits present in the active and passive joints constrain the possible poses of the platform. We next define the equations modelling these constraints, so as to obtain a system of equations describing the workspace $\mathcal{W}$ of the manipulator (Section 2.1). This system is then extended with an additional constraint, in order to select the points that lie on the boundary of $\mathcal{W}$ only (Section 2.2).

\subsection{Workspace Equations}

Let $\mathbf{a}_{i}$ and $\mathbf{b}_{i}$ denote the position vectors of the anchor points $A_{i}$ and $B_{i}$ of the $i$ th leg, respectively expressed in the fixed and moving reference frames. The squared leg lengths can then be written as

$$
l_{i}^{2}=\left|\mathbf{q}_{i}\right|^{2},
$$

for $i=1, \ldots, 6$, where $\mathbf{q}_{i}=\mathbf{p}+\mathbf{R} \mathbf{b}_{i}-\mathbf{a}_{i}$ is a vector aligned with the $i$ th leg, expressed in the fixed reference frame.

Although the entries of $\mathbf{R}$ are here assumed to be variables, they are not independent, since they must define an orthogonal matrix of positive determinant. Such a constraint can be defined in a variety of ways, e.g. by establishing appropriate dot- and cross-product equations on the columns of $\mathbf{R}$, but more intuitive representations of the orientation are obtained when three-parameter expressions for $\mathbf{R}$ are introduced. For ease of comparison with [19], we will here adopt the parameterization provided by roll $(\phi)$, pitch $(\theta)$, and yaw $(\psi)$ angles, for which

$$
\mathbf{R}=\mathbf{R}_{Z}(\psi) \mathbf{R}_{Y}(\theta) \mathbf{R}_{X}(\phi),
$$


or, in columnwise form,

$$
\begin{aligned}
& {\left[\begin{array}{l}
r_{1, x} \\
r_{1, y} \\
r_{1, z}
\end{array}\right]=\left[\begin{array}{c}
\cos \theta \cos \psi \\
\cos \theta \sin \psi \\
-\sin \theta
\end{array}\right],} \\
& {\left[\begin{array}{l}
r_{2, x} \\
r_{2, y} \\
r_{2, z}
\end{array}\right]=\left[\begin{array}{c}
\sin \phi \sin \theta \cos \psi-\cos \phi \sin \psi \\
\sin \phi \sin \theta \sin \psi+\cos \phi \cos \psi \\
\sin \phi \cos \theta
\end{array}\right],} \\
& {\left[\begin{array}{l}
r_{3, x} \\
r_{3, y} \\
r_{3, z}
\end{array}\right]=\left[\begin{array}{c}
\cos \phi \sin \theta \cos \psi+\sin \phi \sin \psi \\
\cos \phi \sin \theta \sin \psi-\sin \phi \cos \psi \\
\cos \phi \cos \theta
\end{array}\right],}
\end{aligned}
$$

but the presented method is applicable to other parameterizations as well, including those based on tilt-and-torsion angles or Euler-Rodrigues parameters.

To see the constraints introduced by the mechanical limits on all joints, note first that the prismatic joints usually have a range of actuation that force the lengths $l_{i}$ to take values within some intervals $\left[l_{i}^{\min }, l_{i}^{\max }\right]$. These constraints can be modelled as equalities by defining $m_{i}=\left(l_{i}^{\max }+l_{i}^{\min }\right) / 2$ and $h_{i}=\left(l_{i}^{\max }-l_{i}^{\min }\right) / 2$, and imposing

$$
\left(l_{i}-m_{i}\right)^{2}+d_{i}^{2}=h_{i}^{2}
$$

for $i=1, \ldots, 6$, where the $d_{i}$ are newly-defined auxiliary variables. Observe that, in this way, the values of $l_{i}$ are certainly constrained to the desired interval, because $m_{i}$ and $h_{i}$ are the mid-point and half-range of the interval, and Eq. (5) defines a circle of center $m_{i}$ and radius $h_{i}$ (Fig. 2).

The passive joints on the base and on the platform also have mechanical limits constraining the allowable positions of each leg. To describe these constraints, let $\mathbf{j}_{A_{i}}$ be a unit vector given in the fixed frame, aligned with the fixed axis of the universal joint at $A_{i}$ (Fig. 3). Following [13], we assume that the mechanical design of such joint restricts the maximum allowed misalignment between $\mathbf{j}_{A_{i}}$ and the $\mathbf{q}_{i}$ vector to be of angle $\alpha_{i}$, which means that, for the platform pose to be valid, it must be

$$
\mathbf{j}_{A_{i}} \mathbf{q}_{i} \geq l_{i} \cos \alpha_{i}, \quad i=1, \ldots, 6
$$

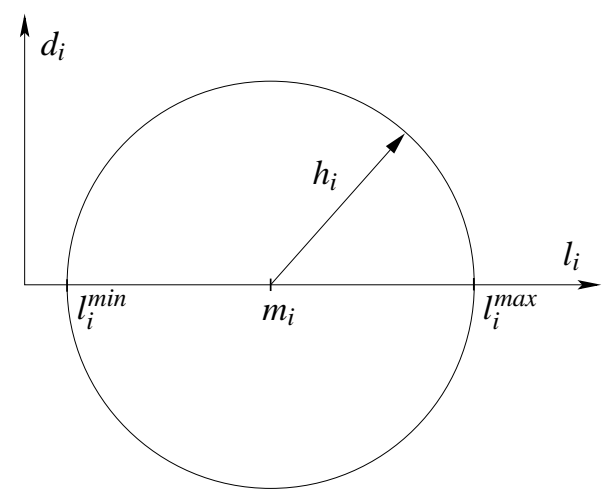

Fig. 2. Circle constraint of an active-joint limit.

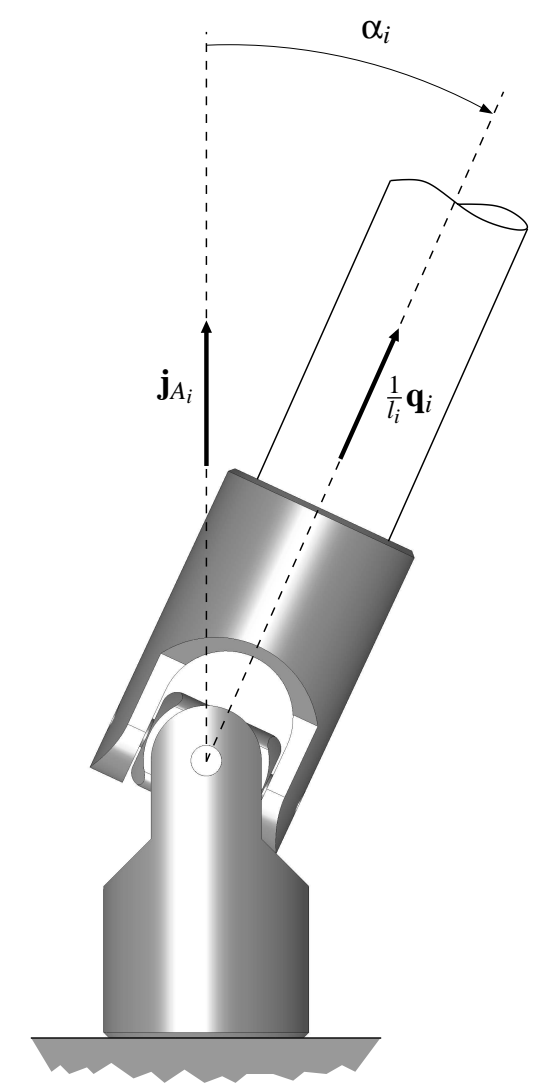

Fig. 3. Elements of a passive joint-limit constraint.

These inequalities can be transformed into equalities by introducing a new variable $t_{i}$ for each leg, and writing

$$
\mathbf{j}_{A_{i}} \mathbf{q}_{i}-l_{i} \cos \alpha_{i}=t_{i}^{2}
$$

Note that $\mathbf{j}_{A_{i}} \mathbf{q}_{i} \geq l_{i} \cos \alpha_{i}$ is satisfied if, and only if, Eq. (6) is satisfied for some value of $t_{i}$. Similarly, for each passive joint on the platform we define a new variable $g_{i}$ and impose

$$
\mathbf{j}_{B_{i}}\left(\mathbf{R}^{\top} \mathbf{q}_{i}\right)-l_{i} \cos \beta_{i}=g_{i}^{2}
$$

where $\mathbf{j}_{B_{i}}$ is a unit vector along the axis of symmetry of the joint at $B_{i}$, expressed in the moving frame, and $\beta_{i}$ is the maximum allowed misalignment in this joint.

In conclusion, the workspace $\mathcal{W}$ of the platform is the set of all possible tuples

$$
(x, y, z, \phi, \theta, \psi)
$$

that satisfy Eqs. (1)-(7) for some value of

$$
\left(l_{1}, \ldots, l_{6}, r_{1, x}, \ldots, r_{3, z}, d_{1}, \ldots, d_{6}, t_{1}, \ldots, t_{6}, g_{1}, \ldots, g_{6}\right)
$$

Since Eqs. (1)-(7) form a system of 33 equations in 39 variables, $\mathcal{W}$ will be a six-dimensional set in general, which is in agreement with the fact that the Stewart platform has six degrees of freedom. 


\subsection{Boundary equations}

We could now try to find $\mathcal{W}$ by solving Eqs. (1)-(7) directly, but it is preferable to compute the boundary of $\mathcal{W}$ instead, because such boundary is a more informative set of lower dimension. A point lies on the boundary of $\mathcal{W}$ whenever any of the active or passive joints reaches a mechanical limit of the joint.

In order to select only the points on the boundary, note that the $i$ th leg reaches its maximal or minimal length, or a limit angle on its passive joints, whenever $d_{i}, t_{i}$, or $g_{i}$ vanish for some $i$. Thus, a solution to Eqs. (1)-(7) corresponds to a boundary point if, and only if,

$$
\prod_{i=1}^{6} d_{i} t_{i} g_{i}=0
$$

In summary, the boundary of $\mathcal{W}$ is formed by the points $(x, y, z, \phi, \theta, \psi)$ that satisfy Eqs. (1)-(8) for some value of the remaining variables. Such points will form a fivedimensional set in general, because just one equation and no extra variable have been added to Eqs. (1)-(7). Despite the boundary of $\mathcal{W}$ has one dimension less than $\mathcal{W}$, it is still hard to compute it exhaustively. However, by setting three pose variables to a constant value, one can obtain twodimensional slices of the boundary whose computation is affordable, as shown below, and yet provide useful representations. Note that, while previous methods concentrate on the constant-orientation or the constant-position slices, any other slice defined by fixing any three of the six pose parameters should also be available to the designer. In our case, such slices are simply characterized by the system of equations formed by Eqs. (1)-(8) with the corresponding three pose variables held constant. The case of the planarmode workspace, where the platform moves on a plane and is only free to rotate about an axis orthogonal to the plane, as well as the constant-orientation and the constant-position workspaces, will be given later as examples of such slices.

\section{Boundary isolation}

A numerical method able to solve the system of equations just described is next provided, adapting the linear relaxation paradigm proposed in [23,24]. The approach entails algebraizing the equations into a quadratic form (Section 3.1), then computing an initial box that bounds the location of all solutions (Section 3.2), and finally using a branchand-prune method exploiting the quadratic form of the equations, to iteratively isolate all boundary points at the desired resolution (Section 3.3).

\subsection{Equation algebraization}

In order to algebraize the system of equations, two different changes of variables need to be introduced. First, all trigonometric terms of Eqs. (2)-(4) are eliminated by intro- ducing the changes of variables

$$
\begin{aligned}
c_{\tau} & =\cos \tau, \\
s_{\tau} & =\sin \tau,
\end{aligned}
$$

for $\tau \in\{\phi, \theta, \psi\}$. Since the new variables $c_{\tau}$ and $s_{\tau}$ represent the cosine and sine of $\tau$, they must obey the circle equations

$$
c_{\tau}^{2}+s_{\tau}^{2}=1
$$

which hence need to be introduced into the system, for $\tau \in\{\phi, \theta, \psi\}$.

After applying such changes, note that the system formed by Eqs. (1)-(9) is already polynomial. Let $\mathbf{y}$ be a vector containing all of the variables of this system, and let $y_{i}$ and $y_{j}$ refer to any two of the components of $\mathbf{y}$. A second change of variables

$$
\begin{aligned}
& p_{k}=y_{i}^{2}, \\
& b_{k}=y_{i} y_{j},
\end{aligned}
$$

is introduced for all $y_{i} y_{j}$ and $y_{i}^{2}$ monomials intervening in the equations, in order to allow transforming the system into the expanded form

$$
\left.\begin{array}{l}
\boldsymbol{\Lambda}(\mathbf{x})=0 \\
\boldsymbol{\Omega}(\mathbf{x})=0
\end{array}\right\}
$$

where $\mathbf{x}$ is an $n_{x}$-dimensional vector including the original $\mathbf{y}$ variables and the newly-introduced $p_{k}$ and $b_{k}$ ones, $\boldsymbol{\Lambda}(\mathbf{x})=0$ is a collection of linear equations in $\mathbf{x}$, and $\boldsymbol{\Omega}(\mathbf{x})=0$ is a collection of quadratic equations, each of which adopts one of the two forms

$$
\begin{aligned}
& x_{k}=x_{i}^{2}, \\
& x_{k}=x_{i} x_{j},
\end{aligned}
$$

which correspond to the changes of variable in Eqs. (10) and (11), respectively. Note that in some cases, as in Eq. (8), the change relative to Eq. (11) needs to be applied recursively in order to arrive at the form assumed in Eq. (12).

\subsection{Initial bounding box}

We next show that each variable $x_{i}$ of $\mathbf{x}$ can only take values within a limited interval, so that from the Cartesian product of all such intervals one can define an $n_{x}$-dimensional box $\mathcal{B} \subset \mathbb{R}^{n_{x}}$ that initially bounds all solutions of Eq. (12).

Since the feasibility intervals for the $p_{k}$ and $b_{k}$ variables can be readily obtained by propagating the intervals of the y variables through Eqs. (10) and (11), we only need to see how to define proper intervals for the $\mathbf{y}$ variables. To this 
end, note that the lengths of the legs are directly bound by their maximal and minimal values, i.e.,

$$
l_{i} \in\left[l_{i}^{\min }, l_{i}^{\max }\right],
$$

for $i=1, \ldots, 6$. Moreover, since the columns of $\mathbf{R}$ are orthonormal vectors, the entries of $\mathbf{R}$ must satisfy

$$
r_{j, x}, r_{j, y}, r_{j, z} \in[-1,1]
$$

for $j=1,2,3$. Similarly, it must be

$$
c_{\tau}, s_{\tau} \in[-1,1]
$$

for $\tau \in\{\phi, \theta, \psi\}$, since these variables refer to the cosine and sine of $\tau$.

For the variables $d_{i}$ we note that Eq. (5) simply constrains such variables to take values in $\left[-h_{i}, h_{i}\right]$. Similarly, Eqs. (6) and (7) constrain the left-hand side of the equation and the $t_{i}$ and $g_{i}$ variables to take values on a parabola of vertical axis with its minimum point at the origin. Note that, in any case, the maximum value that the left-hand side of these equations can achieve is $l_{i}^{\max }-l_{i}^{\max } \cos \alpha_{i}$ or $l_{i}^{\max }-l_{i}^{\max } \cos \beta_{i}$, respectively. In sum, we obtain the following bounds for $i=1, \ldots, 6$ :

$$
\begin{aligned}
& d_{i} \in\left[-h_{i}, h_{i}\right], \\
& t_{i} \in\left[-\sqrt{l_{i}^{\text {max }}-l_{i}^{\text {max }} \cos \alpha_{i}}, \sqrt{l_{i}^{\text {max }}-l_{i}^{\text {max }} \cos \alpha_{i}}\right], \\
& g_{i} \in\left[-\sqrt{l_{i}^{\text {max }}-l_{i}^{\text {max }} \cos \beta_{i}}, \sqrt{l_{i}^{\text {max }}-l_{i}^{\text {max }} \cos \beta_{i}}\right] .
\end{aligned}
$$

Finally, it only remains to find the ranges for the $x, y$, and $z$ components of $\mathbf{p}$. While tight bounds could be computed to accurately confine these variables, this is not necessary under the presented approach. The method is not significantly sensitive to the volume of the initial box because parts with no solution are rapidly discarded by the numerical procedure. Thus, we can use any easy-to-compute upper bound to establish the ranges of these position variables. Here, we shall use the fact that, for any valid configuration of the platform, point $P$ will always lie inside a sphere of radius $l_{i}^{\max }+\left|\mathbf{b}_{i}\right|$ centered at $A_{i}$, which is valid for any leg $i=1, \ldots, 6$. By choosing any leg, for instance leg one, we can take the ranges of the smallest box containing the sphere, that is

$$
\begin{aligned}
& x \in\left[a_{1, x}-l_{1}^{\max }-\left|b_{1}\right|, a_{1, x}+l_{1}^{\max }+\left|b_{1}\right|\right], \\
& y \in\left[a_{1, y}-l_{1}^{\max }-\left|b_{1}\right|, a_{1, y}+l_{1}^{\max }+\left|b_{1}\right|\right], \\
& z \in\left[a_{1, z}-l_{1}^{\max }-\left|b_{1}\right|, a_{1, z}+l_{1}^{\max }+\left|b_{1}\right|\right] .
\end{aligned}
$$

\subsection{Numerical solution}

The algorithm for solving Eq. (12) recursively applies two operations on $\mathcal{B}$ : box shrinking and box splitting. Using box shrinking, portions of $\mathcal{B}$ containing no solution are (a)

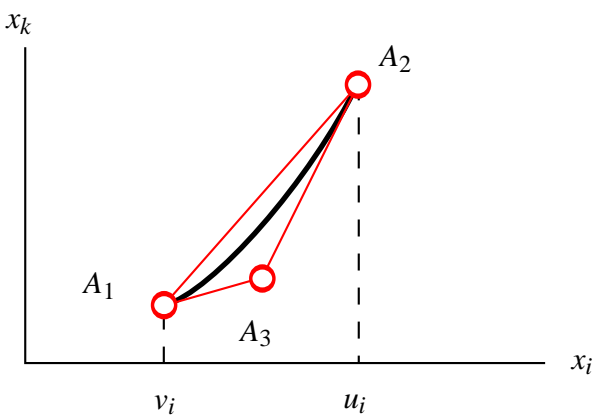

(b)

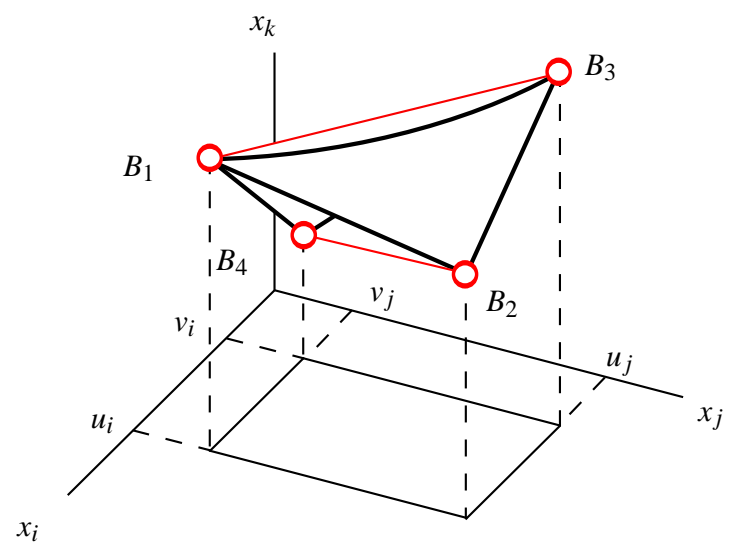

Fig. 4. Polytope bounds within box $\mathcal{B}_{c}$.

eliminated by narrowing some of its defining intervals. This process is repeated until either the box is reduced to an empty set, in which case it contains no solution, or the box is "sufficiently" small, in which case it is considered a solution box, or the box cannot be "significantly" reduced, in which case it is bisected into two sub-boxes via box splitting (which simply bisects its largest interval). To converge to all solutions, the whole process is recursively applied to the new sub-boxes, until one obtains a collection of solution boxes whose side lengths are below a given threshold $\sigma$.

The crucial operation in this scheme is box shrinking, which is implemented as follows. Note first that the solutions falling in some sub-box $\mathcal{B}_{c} \subseteq \mathcal{B}$ must lie in the linear variety defined by $\boldsymbol{\Lambda}(\mathbf{x})=0$. Thus, we may shrink $\mathcal{B}_{c}$ to the smallest possible box bounding this variety inside $\mathcal{B}_{c}$. The limits of the shrunk box along, say, dimension $x_{i}$ can be found by solving the two linear programs

$$
\begin{aligned}
& \text { LP1: } \text { Minimize } x_{i}, \\
& \text { subject to: } \boldsymbol{\Lambda}(\mathbf{x})=0, \mathbf{x} \in \mathcal{B}_{c}, \\
& \text { LP2: } \text { Maximize } x_{i}, \\
& \text { subject to: } \boldsymbol{\Lambda}(\mathbf{x})=0, \mathbf{x} \in \mathcal{B}_{c} .
\end{aligned}
$$

However, observe that $\mathcal{B}_{c}$ can be further reduced, because the solutions must also satisfy all equations $x_{k}=x_{i}^{2}$ and $x_{k}=x_{i} x_{j}$ in $\boldsymbol{\Omega}(\mathbf{x})=0$. These equations can be taken into account by noting that, if $\left[v_{i}, u_{i}\right]$ denotes the interval of $\mathcal{B}_{c}$ along dimension $x_{i}$, then: 
1. The portion of the parabola $x_{k}=x_{i}^{2}$ lying inside $\mathcal{B}_{c}$ is bound by the triangle $A_{1} A_{2} A_{3}$, where $A_{1}$ and $A_{2}$ are the points where the parabola intercepts the lines $x_{i}=v_{i}$ and $x_{i}=u_{i}$, and $A_{3}$ is the point where the tangent lines at $A_{1}$ and $A_{2}$ meet (Fig. 4a).

2. The portion of the hyperbolic paraboloid $x_{k}=x_{i} x_{j}$ lying inside $\mathcal{B}_{c}$ is bound by the tetrahedron $B_{1} B_{2} B_{3} B_{4}$, where the points $B_{1}, \ldots, B_{4}$ are obtained by lifting the corners of the rectangle $\left[v_{i}, u_{i}\right] \times\left[v_{j}, u_{j}\right]$ vertically to the paraboloid (Fig. 4b).

Thus, linear inequalities corresponding to these bounds can be added to the previous linear programs LP1 and LP2, which usually produces a much larger reduction of $\mathcal{B}_{c}$ or, if one of the linear programs is found unfeasible, its complete elimination.

As it turns out, the previous algorithm explores a binary tree of boxes whose internal nodes correspond to boxes that have been split at some time, and whose leaves are either solution or empty boxes. The collection $B$ of all solution boxes, which is returned as output upon termination, is said to form a box approximation of the solution set of Eq. (12), because the boxes in $B$ form a discrete envelope of such set, whose accuracy can be adjusted through the $\sigma$ parameter. Notice that the algorithm is complete, in the sense that it will succeed in isolating all solution points of Eq. (12) accurately, provided that a small-enough value for $\sigma$ is used. Detailed properties of the algorithm, including an analysis of its completeness, correctness, and convergence order, are given in [24].

\section{Illustrative examples}

We next illustrate the performance of the method by computing the boundaries of several workspaces of the Stewart platform. To emphasize the generality of the method, we first obtain the boundaries corresponding to the constantorientation, constant-position and planar-mode workspaces of a standard platform. We then analyze a special platform to show that the method performs well on situations that hinder the application of previous methods. The method is able to completely determine the boundaries of the workspace in all situations.

The geometric parameters of the analyzed platforms are indicated in Table 1, and the ranges for $l_{i}$ are assumed to be $[1.2,1.8]$ for all legs. All results reported have been obtained by using a parallelized version of the method, implemented in $\mathrm{C}$ using previous software libraries developed in [23,24], and executed on a grid computer with four PC units equipped with two Intel Quadcore Xeon E5310 processors and 4 Gb of RAM each one. Table 2 provides, for each experiment, the amount of CPU time required to solve it $\left(t_{s}\right)$ and the number of solution boxes returned $\left(n_{s}\right)$ using $\sigma=0.1$.

\subsection{A standard platform}

The standard platform analyzed here corresponds to the one studied in [19], where the authors compute the constantposition workspace obtained by fixing $\mathbf{p}$ to a constant value, but neglecting the mechanical limits on the passive joints.
Table 1. Stewart platform parameters.

\begin{tabular}{|c|c|c|c|c|c|c|c|}
\hline & $i$ & 1 & 2 & 3 & 4 & 5 & 6 \\
\hline \multirow{6}{*}{ 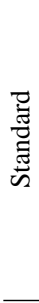 } & $a_{i, x}$ & 0 & 0 & 0.7598 & 0.7598 & -0.7598 & -0.7598 \\
\hline & $a_{i, y}$ & 0 & 0 & 1.3161 & 1.3161 & 1.3161 & 1.3161 \\
\hline & $a_{i, z}$ & 0 & 0 & 0 & 0 & 0 & 0 \\
\hline & $b_{i, x}$ & -0.4559 & 0.4559 & 0.4599 & 0 & 0 & -0.4559 \\
\hline & $b_{i, y}$ & -0.2632 & -0.2632 & -0.2632 & 0.5264 & 0.5264 & -0.2632 \\
\hline & $b_{i, z}$ & 0 & 0 & 0 & 0 & 0 & 0 \\
\hline \multirow{6}{*}{ 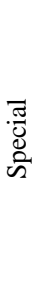 } & $a_{i, x}$ & 0.8165 & -0.4082 & -0.4082 & 0.8165 & -0.4082 & -0.4082 \\
\hline & $a_{i, y}$ & 0 & 0.7071 & -0.7071 & 0 & 0.7071 & -0.7071 \\
\hline & $a_{i, z}$ & 0 & 0 & 0 & 0 & 0 & 0 \\
\hline & $b_{i, x}$ & 0 & 0 & 0 & 0.8165 & -0.4082 & -0.4082 \\
\hline & $b_{i, y}$ & 0 & 0 & 0 & 0 & 0.7071 & -0.7071 \\
\hline & $b_{i, z}$ & 0 & 0 & 0 & 0.5774 & 0.5774 & 0.5774 \\
\hline
\end{tabular}

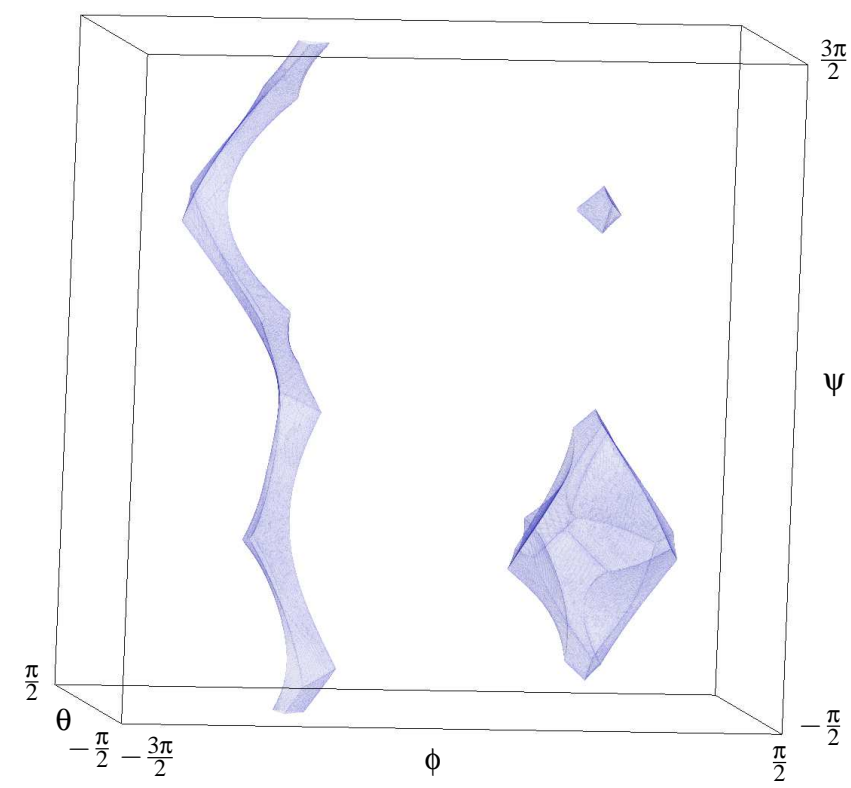

Fig. 5. Boundaries of the constant-position workspace of the standard platform, without taking passive joint limits into account.

To see that such limits really reduce the workspace, we have computed the workspace twice: first neglecting the limits on the passive joints, and then taking them into account. Fig. 5 shows the obtained results for the roll-pitch-yaw angles, assuming $\mathbf{p}=\left[0, \frac{2}{3} \sqrt[4]{3}, \frac{5}{4}\right]^{\top}$ as in [19]. As it can be seen, the workspace contains three different connected components, while in [19] the authors are only able to describe the one accessible from the origin (the lower-right component in Fig. 5). Detecting all connected components is necessary though, to let the designer choose the most appropriate assembly mode for the platform, depending on the specific task to be performed.

By taking into account the limit angles $\alpha_{i}=\beta_{i}=50^{\circ}$ on all passive joints, some of the components in Fig. 5 are no longer accessible, and the workspace reduces to just one 

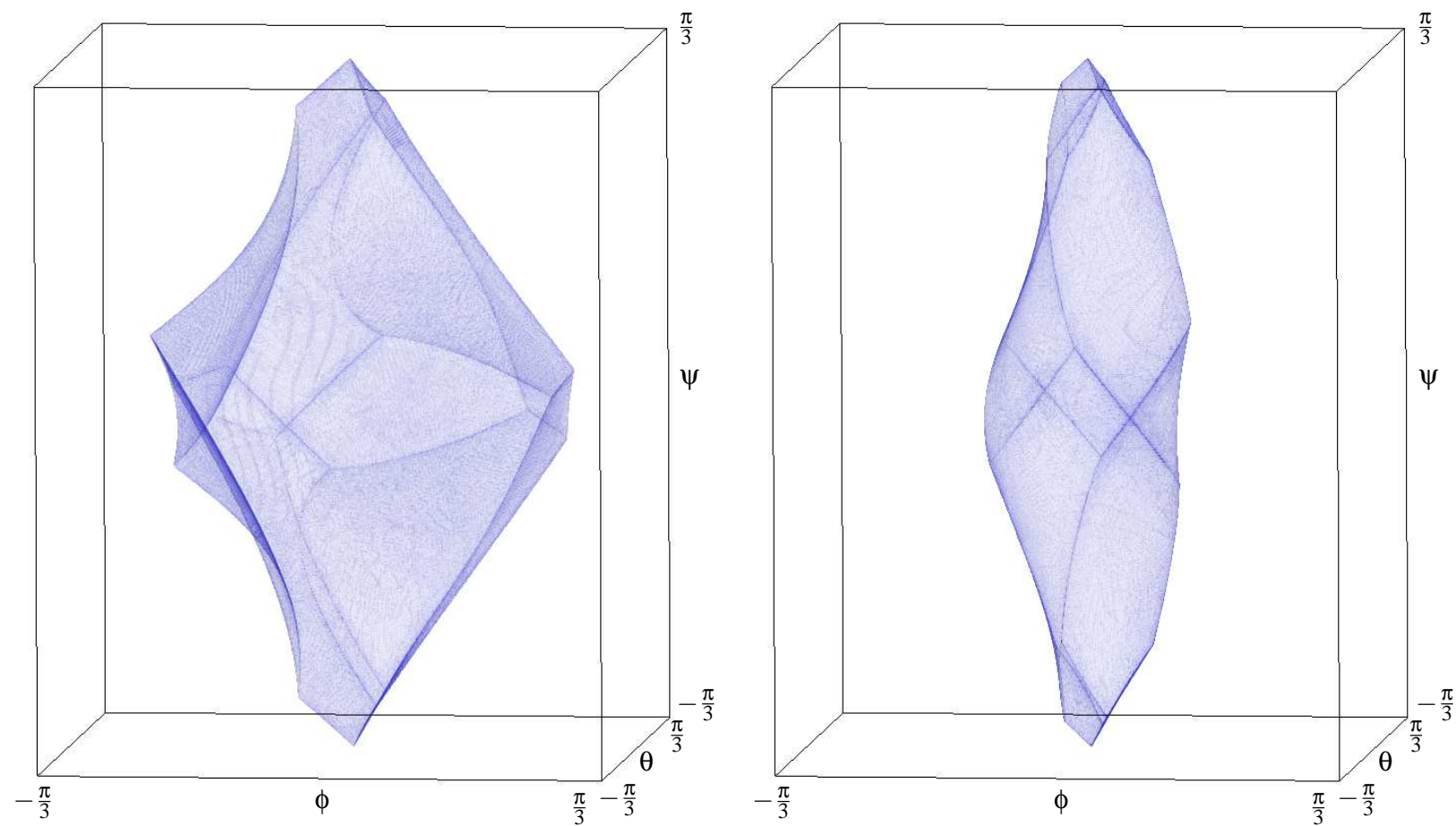

Fig. 6. Only the lower-right component in Fig. 5 (left) is partially achievable after taking passive-joint limits into account (right).
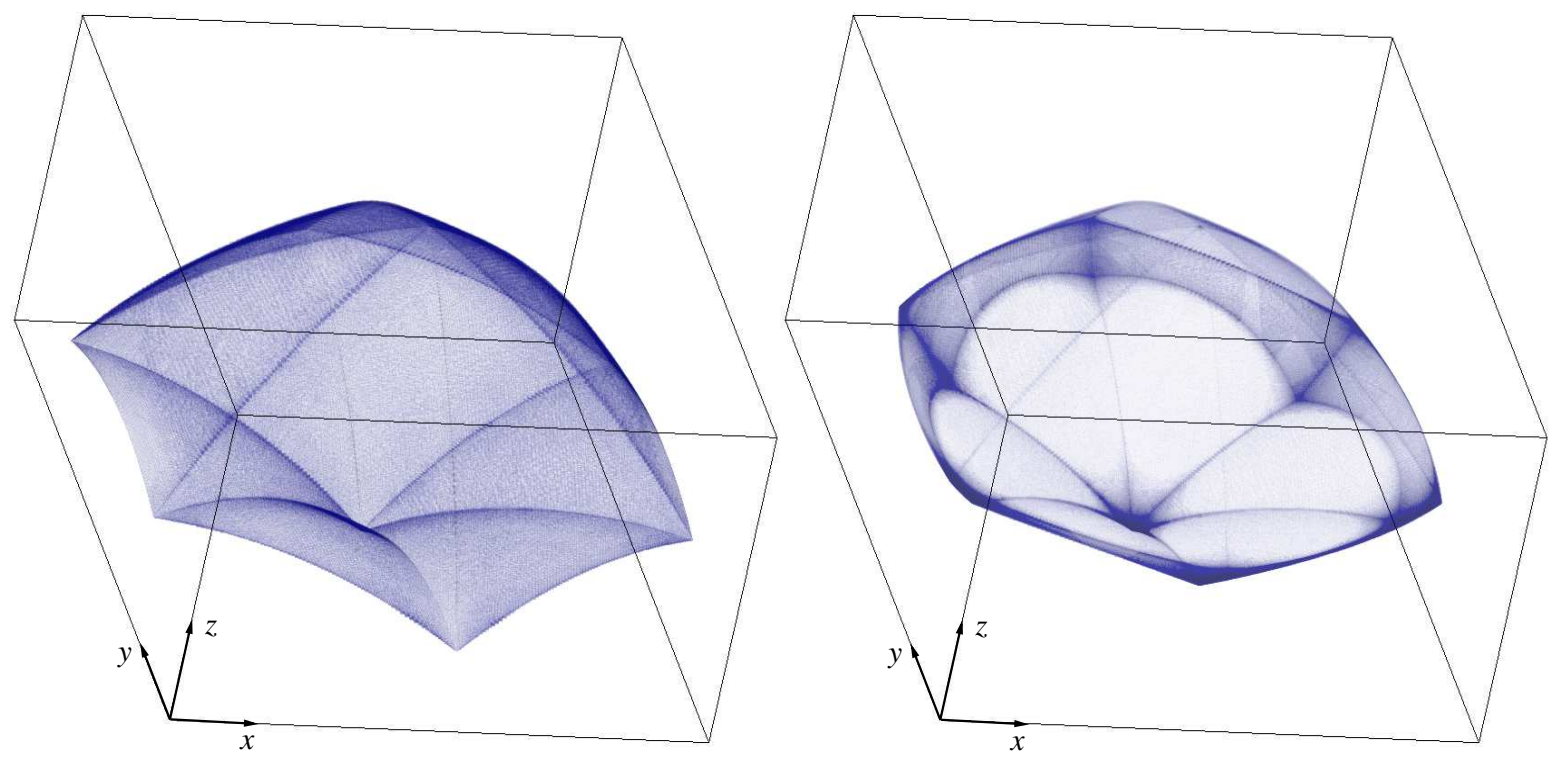

Fig. 7. Boundaries of the constant-orientation workspace of the standard platform for $z>0$.

connected component that corresponds to the one around the origin in Fig. 5. For comparison, Fig. 6 shows such component before and after the consideration of passive-joint-limit constraints.

Instead of fixing $\mathbf{p}$, we can fix the orientation of the platform, and hence obtain the constant-orientation workspace. Setting $\phi=\theta=\psi=0$, which is equivalent to letting $\mathbf{R}=\mathbf{I}_{3}$, we obtain the workspace shown in Fig. 7. The figure compares the resulting workspaces when taking into account only active-joint-limit constraints (left) or active- and passive-joint-limit constraints simultaneously (right), with $\alpha_{i}=\beta_{i}=47.16^{\circ}$. As before, the enclosed volume is smaller in the second case, meaning that the attainable positions of the platform are reduced because some of the passive joints achieve their mechanical limits. It is worth adding, also, that in both cases the workspace has an additional connected component symmetric to the one shown, which corresponds to the assembly mode of the platform where P sweeps a similar volume for $z<0$.

In general, previous methods in the literature only compute discretized versions of the constant-position or the constant-orientation slices of the complete six-dimensional 

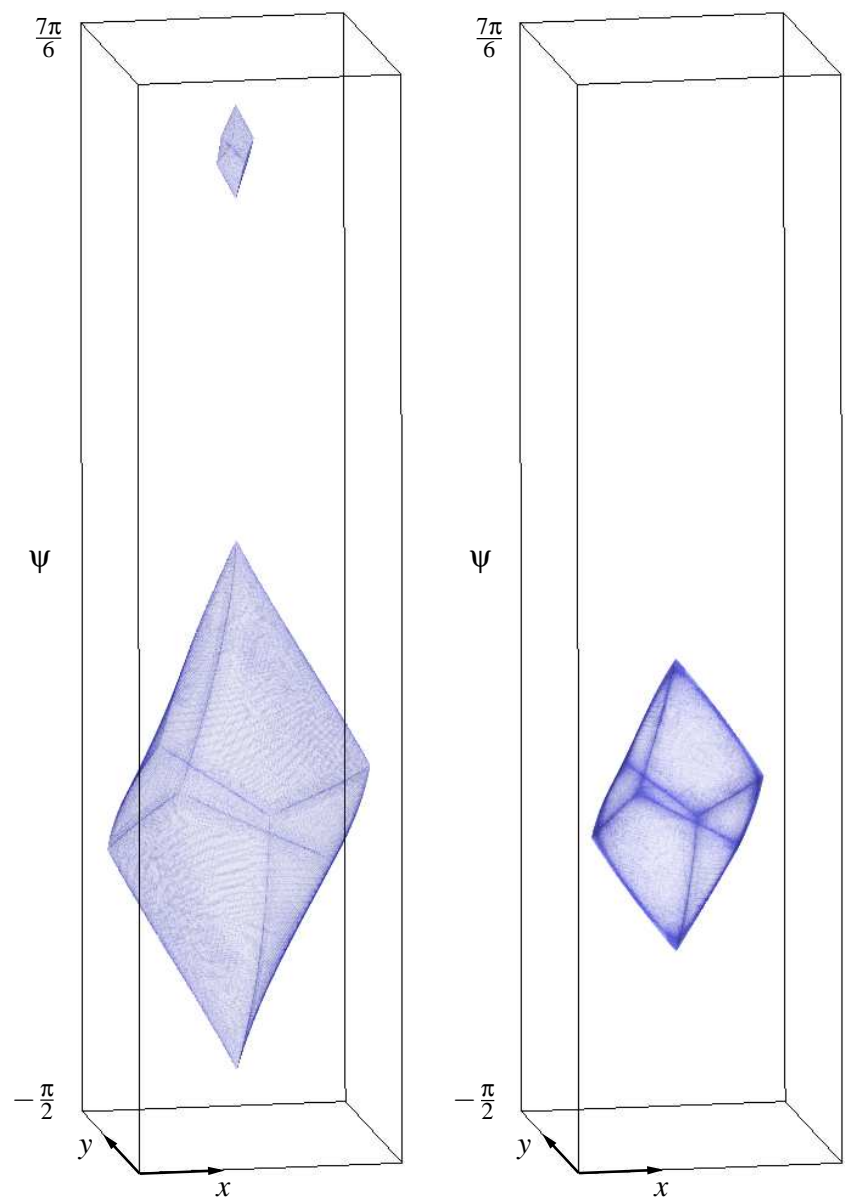

Fig. 8. Boundaries of the planar-mode workspace of the standard platform before and after the consideration of passive-joint limits.

workspace, i.e., those shown so far in Figs. 5-7. But the method herein described can also be used to derive any other slice. As an example, Fig. 8 presents the slices obtained when fixing $z=\frac{5}{4}$ and $\phi=\theta=0$, before and after the consideration of passive joint limits with $\alpha_{i}=\beta_{i}=41.41^{\circ}$. Note that in this case the platform is equivalent to a planar positioning device, where $P$ moves on the $z=\frac{5}{4}$ plane, and only rotation about the $Z^{\prime}$ axis is allowed.

\subsection{A special platform}

We next show results on computing the constantposition workspace of a special platform that yields interior barriers in such workspace. Its geometric parameters are shown in Table 1 and correspond to a design where three of the legs are anchored at a same point $P$ on the platform, with the base joints coincident in pairs. This design may seem difficult to construct, but if $P$ is the point we keep fixed when computing the constant-position workspace, then the platform is equivalent to the 3-UPS/S manipulator shown in Fig. 9, which allows orientational capability only, and is in fact kinematically equivalent to the Agile Eye platform [25].

While on general platforms the constant-position workspace has a two-dimensional boundary, such boundary degenerates into a one-dimensional set in this case, as shown

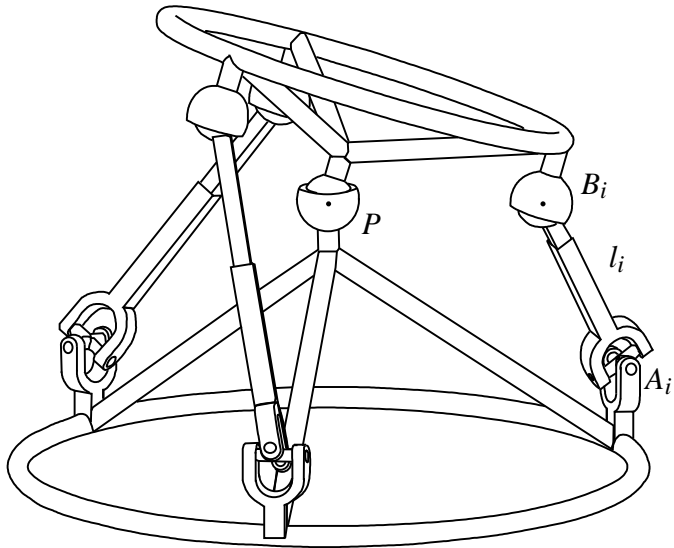

Fig. 9. A 3-UPS/S platform. (Figure adapted from [25].)

in Fig. 10, and this set is impossible to obtain by previous numerical methods. The method in [16], for example, would proceed by intersecting the boundaries through planes, and then computing the portion of the boundaries lying in such planes using randomized ray-shooting techniques combined with numerical path-following methods. However, note that the planes would only contain isolated points in this case, which would almost never be hit by rays shot in random directions. Also, the method in [19] would perform a discretization on angle $\theta$ and then on angle $\phi$ in order to find the boundary points corresponding to such angles. Nevertheless, the portion of a slice lying on a constant $\theta$ plane is again formed by isolated points, and a discretization on angle $\phi$ would almost never encounter such points. So far, actually, slices of the boundary curves in Fig. 10 could only be obtained by analytical methods tailored to such platforms [25], because other methods based on discretization exhibit similar drawbacks $[13,15,17]$. The presented technique, on the contrary, is robust to such situations. The results shown in Fig. 10 were obtained by solving the same equations considered for the standard platform, by changing the platform parameters only. As noted from the results, the workspace of this manipulator is the whole range of the roll-pitch-yaw angles, but the shown curves represent motion impediments to be avoided by the platform. In other words, the manipulator will be able to reach any possible orientation, but it will be unable to traverse across the curves.

\section{Conclusions}

This paper has introduced a new approach for computing three-dimensional slices of the workspace on Stewart platforms of arbitrary geometry. A distinguishing feature of the approach is that it allows, using a single method, the obtention of any possible slice of the workspace, while previous approaches mostly concentrate on particular slices, like the constant-position or the constant-orientation slice. In fact, a total of twenty slices can be obtained by fixing three pose parameters, and computing any of them might be necessary depending on the specific task to be performed with the plat- 


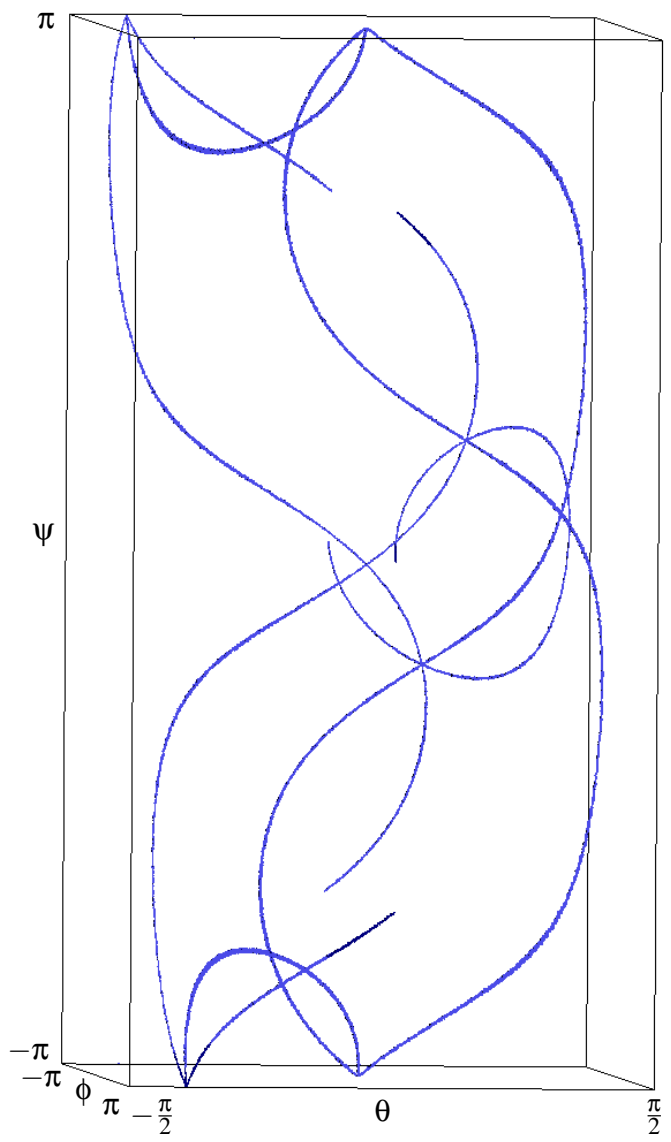

Fig. 10. Degenerate boundaries of the constant-position workspace of the special platform.

Table 2. Performance data at $\sigma=0.1$.

\begin{tabular}{ccccc} 
Platform & Workspace & Joint limits & $n_{s}$ & $t_{s}(\mathrm{sec})$ \\
\hline \multirow{4}{*}{ Standard } & \multirow{2}{*}{ Const. position } & Active & 6526 & 66 \\
& \multirow{2}{*}{ Const. orientation } & Active and passive & 3196 & 69 \\
\cline { 2 - 5 } & & Active & 1306 & 4 \\
& \multirow{2}{*}{ Planar mode } & Active and passive & 1621 & 21 \\
\hline \multirow{2}{*}{ Special } & Const. position & Active & 1849 & 6 \\
\hline & & Active and passive & 1391 & 14 \\
\hline & & & 8448 & 252 \\
\hline
\end{tabular}

form. Additional advantages of the method have been discussed and illustrated with examples, like the ability to compute all connected components of a slice, to detect motion barriers present in its interior, and the possibility to take passive joint limits into account.

Clearly, the primary application of the method is in the context of robot design, because it allows studying the motion capability of a particular platform before its actual construction. However, the method might also be helpful in the context of collision-free trajectory planning, where a main issue is how to sample the workspace efficiently and with good coverage, in order to compute proper roadmaps of the workspace in short times [8]. While fast in favorable situations, current planners draw samples from conservative estimates of the workspace, which makes them less efficient on highly-constrained situations. The performance of such planners would notably increase if samples were drawn from accurate representations like those provided by the proposed method.

Finally, it is worth noting that, as shown in [27, 28], a similar approach can be applied to also compute the forward singularities contained in the workspace of the platform, and that the presented method also shows some potential of being able to cope with leg-leg collision constraints. The latter constraints could in principle be formulated in the form required by the numerical method, but further work needs to be done in order to achieve a mild formulation involving as few equations and variables as possible, leading to an acceptable computational burden.

\section{Achnowledgements}

The authors are grateful to Dr. Josep M. Porta for fruitful discussions around the topic of the paper, and for his help during the implementation of the method. This work has been partially supported by the Spanish Ministry of Science and Innovation under contract DPI2010-18449, and under a Juan de la Cierva contract supporting the second author.

\section{References}

[1] Stewart, D., 1965. "A platform with six degrees of freedom". Proc. of the Institution of Mechanical Engineers, 180, pp. 371-386.

[2] McInroy, J. E., and O’Brien, G. W., 1999. "Precise, fault-tolerant precision pointing using a Stewart platform". IEEE/ASME Transactions on Mechatronics, 4, pp. 91-95.

[3] van Silfhout, R. G., 1999. "High-precision hydraulic Stewart platform". Review of Scientific Instruments, 70, pp. 3488-3494.

[4] Preumont, A., Horodinca, M., Romanescu, I., de Marneffe, B., Avraam, M., Deraemaeker, A., Bossens, F., and Hanieh, A., 2007. "A six-axis single-stage active vibration isolator based on Stewart platform". Journal of Sound and Vibration, 300, pp. 644-661.

[5] Shaw, D., and Chen, Y., 2001. "Cutting path generation of the Stewart platform-based milling machine using an end-mill". International Journal of Production Research, 39, pp. 1367-1383.

[6] Pashkevich, A., Chablat, D., and Wenger, P., 2006. "Kinematics and workspace analysis of a three-axis parallel manipulator: the orthoglide". Robotica, 24(1), pp. 39-50.

[7] Shoham, M., Burman, M., Zehavi, E., Joskowicz, L., Batkilin, E., and Kunicher, Y., 2003. "Bone-mounted miniature robot for surgical procedures: Concept and clinical applications". IEEE Transactions on Robotics and Automation, 19(5), october. 
[8] Cortés, J., and Siméon, T., 2003. "Probabilistic motion planning for parallel mechanisms". In Proc. of the International Conference on Robotics and Automation, Vol. 3, pp. 4354-4359.

[9] Dasgupta, B., and Mruthyunjaya, T. S., 2000. “The Stewart platform manipulator: a review". Mechanism and Machine Theory, 35(1), pp. 15-40.

[10] Gosselin, C., 1990. "Determination of the workspace of 6-DOF parallel manipulators". ASME Journal of Mechanical Design, 112, pp. 331-336.

[11] Merlet, J. P., 1992. "Geometrical determination of the workspace of a constrained parallel manipulator". In Advances in Robot Kinematics, Ferrare, Italy, September 7-9, pp. 326-329.

[12] Merlet, J.-P., et al., 1999. "Determination of 6D workspaces of Gough-type parallel manipulator and comparison between different geometries". International Journal of Robotics Research, 18(9), pp. 902916.

[13] Bonev, I., and Ryu, J., 2001. "A new approach to orientation workspace analysis of 6-DOF parallel manipulators". Mechanism and Machine Theory, 36(1), pp. 1528.

[14] Romdhane, L., 1994. "Orientation workspace of fully parallel mechanisms". European Journal of Mechanics, A/Solids, 13(4), pp. 541-553.

[15] Merlet, J.-P., 1995. "Determination of the orientation workspace of parallel manipulators". Journal of Intelligent and Robotic Systems, 13(2), pp. 143-160.

[16] Haug, E. J., Luh, C.-M., Adkins, F. A., and Wang, J.Y., 1996. "Numerical algorithms for mapping boundaries of manipulator workspaces". ASME Journal of Mechanical Design, 118, pp. 228-234.

[17] Pernkopf, F., and Husty, M., 2006. "Workspace analysis of Stewart-Gough-type parallel manipulators". Proc. of the Institution of Mechanical Engineers, Part C: Journal of Mechanical Engineering Science, 220(7), pp. 1019-1032.

[18] Tsai, K., and Lin, J., 2006. "Determining the compatible orientation workspace of Stewart-Gough parallel manipulators". Mechanism and Machine Theory, 41(10), pp. 1168-1184.

[19] Jiang, Q., and Gosselin, C., 2009. "Evaluation and representation of the theoretical orientation workspace of the gough-stewart platform". ASME Journal of Mechanisms and Robotics, 1(2).

[20] Luh, C. M., Adkins, F. A., Haug, E. J., and Qiu, C. C., 1996. "Working capability analysis of Stewart platforms". ASME Journal of Mechanical Design, 118, pp. 220-227.

[21] Abdel-Malek, K., Adkins, F., Yeh, H. J., and Haug, E., 1997. "On the determination of boundaries to manipulator workspaces". Robotics and Computer Integrated Manufacturing, 13(1), pp. 63-72.

[22] Bohigas, O., Manubens, M., and Ros, L. "A complete method for workspace boundary determination on general structure manipulators". IEEE Transactions on Robotics. In press.
[23] Porta, J. M., Ros, L., Creemers, T., and Thomas, F., 2007. "Box approximations of planar linkage configuration spaces". ASME Journal of Mechanical Design, 129(4), pp. 397-405.

[24] Porta, J. M., Ros, L., and Thomas, F., 2009. "A linear relaxation technique for the position analysis of multiloop linkages". IEEE Transactions on Robotics, 25(2), pp. 225-239.

[25] Bonev, I. A., and Gosselin, C. M., 2006. "Analytical determination of the workspace of symmetrical spherical parallel mechanisms". IEEE Transactions on Robotics, 22(5), pp. 1011-1017.

[26] Merlet, J., 2006. Parallel robots. Springer-Verlag New York Inc.

[27] Bohigas, O., Zlatanov, D., Ros, L., Manubens, M., and Porta, J. M., 2012. "Numerical computation of manipulator singularities". In Proc. of the International Conference on Robotics and Automation. In press.

[28] Bohigas, O., Zlatanov, D., Manubens, M., and Ros, L., 2012. "On the numerical classification of the singularities of robot manipulators". In Proc. of the ASME International Design Engineering Technical Conferences. In press. 